\title{
Gold Nanorod Bioconjugates for Active Tumor Targeting and Photothermal Therapy
}

\author{
Hadiyah N. Green, ${ }^{1}$ Dmitry V. Martyshkin,, ${ }^{1}$ Cynthia M. Rodenburg, ${ }^{2}$ \\ Eben L. Rosenthal, ${ }^{3}$ and Sergey B. Mirov ${ }^{1}$ \\ ${ }^{1}$ Center for Optical Sensors and Spectroscopies and the Department of Physics, The University of Alabama at Birmingham, \\ Campbell Hall 310, 1300 University Boulevard, Birmingham, AL 35294, USA \\ ${ }^{2}$ Department of Microbiology, The University of Alabama at Birmingham, Bevill Biomedical Research Building, \\ 845 19th Street South, Birmingham, AL 35294, USA \\ ${ }^{3}$ Division of Otolaryngology, Head and Neck Surgery, Department of Surgery, The University of Alabama at Birmingham, \\ Volker Hall G082, 1670 University Boulevard, Birmingham, AL 35233, USA
}

Correspondence should be addressed to Hadiyah N. Green, hadiyahnicole@gmail.com

Received 19 July 2011; Revised 11 August 2011; Accepted 16 August 2011

Academic Editor: Mahi R. Singh

Copyright ( 92011 Hadiyah N. Green et al. This is an open access article distributed under the Creative Commons Attribution License, which permits unrestricted use, distribution, and reproduction in any medium, provided the original work is properly cited.

The mastery of active tumor targeting is a great challenge in near infrared photothermal therapy (NIRPTT). To improve efficiency for targeted treatment of malignant tumors, we modify the technique of conjugating gold nanoparticles to tumorspecific antibodies. Polyethylene glycol-coated (PEGylated) gold nanorods (GNRs) were fabricated and conjugated to an antiEGFR antibody. We characterized the conjugation efficiency of the GNRs by comparing the efficiency of antibody binding and the photothermal effect of the GNRs before and after conjugation. We demonstrate that the binding efficiency of the antibodies conjugated to the PEGylated GNRs is comparable to the binding efficiency of the unmodified antibodies and $33.9 \%$ greater than PEGylated antibody-GNR conjugates as reported by Liao and Hafner (2005). In addition, cell death by NIRPTT was sufficient to kill nearly $90 \%$ of tumor cells, which is comparable to NIRPTT with GNRs alone confirming that NIRPTT using GNRs is not compromised by conjugation of GNRs to antibodies.

\section{Introduction}

One of the greatest obstacles with cancer treatments, past and present, is the ability to actively target and selectively treat only the malignant cells while leaving normal cells unharmed. Near infrared (NIR) photothermal therapy (PTT) using gold nanoparticles (GNPs) and NIR light to treat malignant tumors in vitro and in vivo have demonstrated promise as treatments for cancer [1-11]. The collective concept would lead to a single modality that targeted, imaged, and treated malignant tumors. In general, PTT utilizes a contrast agent in the form of GNPs having plasmon resonance in the NIR spectral range where normal tissue is transparent. The GNPs convert the absorbed NIR light energy into thermal heat energy and cause cell death. Even though the therapeutic properties of PTT have been demonstrated, further development of the active targeting component is needed.

The targeting strategy was the main difference between the in vitro and in vivo studies for some of the successful PTTs performed: the in vitro practice of nanoparticle conjugation to tumor targeting antibodies was not implemented in the in vivo models. For example, it has been shown that $\mathrm{SiO}_{2}$ core gold-shell nanospheres [3] and gold nanorods [4] were conjugated to monoclonal antibodies (Mab) and used for NIR PTT in vitro. The in vivo studies performed by the same research groups utilized unconjugated nanoparticles for PTT $[12,13]$, and the enhanced permeability and retention (EPR) effect due to leaky tumor vasculature was the reason cited for the nanoparticle assembly at the tumor site. However, the EPR effect is a passive targeting strategy allowing stray nanoparticles to disperse throughout the body 
and accumulate in non-tumor-related sites. Active targeting, on the other hand, requires successful GNPs-to-antibody conjugation using covalent bonds to ensure specific delivery to the tumor.

The standard for conjugating gold nanoparticles to antibodies using covalent bonding was published by pioneers in the field, Liao and Hafner [14]. However, a durable conjugation process is needed to protect the conjugation from the physiological conditions of the body, reduce nonspecific binding, ensure active delivery of the nanoparticles to the malignant tumor site, and increase the efficacy of the NIR laser treatment. Incorporating a method of active targeting will improve the impact of PTT making it a viable approach for a variety of carcinomas that overexpress the epidermal growth factor including head and neck, colorectal, ovarian, skin, cervical, breast, bladder, pancreatic, and prostate cancers. In this study, we report a comparison of the Liao and Hafner protocol for PEGylating antibody-GNRs conjugates [14] to our protocol of conjugating antibodies to PEGylated GNRs resulting in a $33.9 \%$ improvement in the conjugation efficiency of GNRs to tumor-targeted antibodies.

\section{Materials and Methods}

2.1. Antibody. Cetuximab (ImClone Systems, New York, NY), a recombinant human/mouse chimeric monoclonal IgG antibody, was used in this study. This monoclonal antibody binds specifically to the extracellular domain of the human EGFR, which is overexpressed in head and neck cancers. Cetuximab is composed of the $\mathrm{Fc}$ regions of a murine anti-EGFR antibody and human immunoglobulin IgG1 heavy and kappa light chain constant regions and has an approximate molecular weight of $152 \mathrm{kDa}$. Cetuximab is supplied as a $2 \mathrm{mg} / \mathrm{mL}$ solution containing sodium chloride, sodium phosphate dibasic heptahydrate, sodium phosphate monobasic monohydrate, in water, and has a $\mathrm{pH}$ ranging from 7.0 to 7.4 .

2.2. Crosslinker. Long Chain Succinimidyl 6-(3-[2-pyridyldithio]-propionamido) hexanoate (LC-SPDP), MW = $425.52 \mathrm{~g} / \mathrm{mol}$, spacer arm length $=15.6$ Angstroms (Thermo Scientific, Rockford, IL), was employed to bioconjugate cetuximab to gold nanorods. LC-SPDP is a heterobifunctional, thiol-cleavable, and membrane permeable crosslinker. LC-SPDP contains an amine-reactive $N$-hydroxysuccinimide (NHS) ester that will react with lysine residues to form a stable amide bond on the surface of the antibody. The other end of the spacer arm is terminated in the pyridyl disulfide group that will react with sulfhydryls to form a reversible disulfide bond that reacts with thiol PEGylated gold nanorods.

2.3. Gold Nanorod Fabrication. Gold nanorods were fabricated according to the method described by Sau and Murphy [15] summarized as follows.

Stock Solutions Preparation. $30 \mathrm{~mL}$ of HPLC grade water was placed on ice. While the temperature of the water was cooling to 0 degrees Celsius, the other solutions were prepared: $1 \mathrm{mM}$ Gold (III) chloride trihydrate, $\left(\mathrm{HAuCl}_{4} \cdot 3 \mathrm{H}_{2} \mathrm{O}\right)$ (Sigma-Aldrich), $200 \mathrm{mM}$ cetyltrimethylammonium bromide (CTAB) (Sigma-Aldrich), $78.8 \mathrm{mM}$ Ascorbic acid, and $32 \mathrm{mM} \mathrm{AgNO}_{3}$ (Sigma-Aldrich). A hot-water bath, less than $50^{\circ} \mathrm{C}$, was used to dissolve $\mathrm{CTAB}$. Ice-cold water was rapidly added to the $\mathrm{NaBH}_{4}$ and the solution was returned to ice since $\mathrm{NaBH}_{4}$ solution is unstable and rapidly decomposes at room temperature.

Seeds Solution Preparation. $2.5 \mathrm{~mL}$ of $1 \mathrm{mM}$ of $\mathrm{HAuCl}_{4}$. $3 \mathrm{H}_{2} \mathrm{O} ; 5 \mathrm{~mL} 200 \mathrm{mM} \mathrm{CTAB}$; and $0.6 \mathrm{~mL}$ ice-cold $10 \mathrm{mM}$ $\mathrm{NaBH}_{4}$ were mixed and incubated at room temperature $\left(25^{\circ} \mathrm{C}\right)$ for 2 hours before use. This last step is needed to disintegrate the remaining $\mathrm{NaBH}_{4}$ to prevent unwanted seeds appearing in the growth solution. The seed solution at this stage should be beige-brown in color.

Growth Solution Preparation. $17-20 \mathrm{~mL}$ of $1 \mathrm{mM} \mathrm{HAuCl}{ }_{4}$. $3 \mathrm{H}_{2} \mathrm{O}$ and $17-20 \mathrm{~mL}$ of $200 \mathrm{mM} \mathrm{CTAB}$ were combined with $80-120 \mu \mathrm{L}$ of $32 \mathrm{mM} \mathrm{AgNO}_{3}, 280-360 \mu \mathrm{L}$ of $78.8 \mathrm{mM}$ Ascorbic acid, and $60-80 \mu \mathrm{L}$ of seed solution. The solution color changed from yellow-orange to colorless and then finally to deep pink. Nanorods were allowed to grow undisturbed for $2 \mathrm{~h}$ at room temperature. The $\mathrm{NaBH}_{4}$ should completely decompose during this stage.

2.4. Gold Nanorod Biofunctionalization Using Polyethylene Glycol (PEG). Gold nanorods were fabricated with the surfactant, $C T A B$, as a capping agent to control the size of the nanorod. The process of using poly-ethylene glycol (PEG) to replace the $\mathrm{CTAB}$ on the surface of nanorods is known as "PEGylation" whereby the nanorods are "PEGylated." PEGylation is advantageous because it increases biocompatibility and stability, decreases immunogenicity and adsorption to the negatively charged luminal surface of blood vessels, and suppresses the nonspecific binding of charged molecules $[5,16,17]$. During the CTAB removal process, the nanorods were centrifuged at $(7000 \mathrm{~g}, 20 \mathrm{~min})$, decanted, and the pellet was resuspended in $2 \mathrm{~mL}$ of $100 \mathrm{mM}$ PBS-EDTA. The nanorods were then biofunctionalized, PEGylated, using $1 \mathrm{mM}$ of thiol-terminated methoxy-poly-ethylene glycol $(\mathrm{mPEG}-\mathrm{SH})(\mathrm{MW}=5000$, Nanocs, New York, NY) and $2 \mathrm{mM}$ of Potassium Carbonate (Acros, Fair Lawn, NJ) and incubated overnight. A covalent bond was formed between the thiol group of PEG and the surface of the gold nanorod replacing the CTAB. A VersaMax microplate reader was used to determine the relative optical density and concentration of the nanorods. The measured concentration of the PEGylated nanorods was CONC $C_{\text {PEG-NR }}=4.41 \mathrm{mg} / \mathrm{mL}$. The optical density of the PEGylated nanorods was OD PEG-NR $=8.24$ $\left(\sim 1.73 \times 10^{12} \mathrm{GNRs} / \mathrm{mL}\right)$ unless otherwise noted. UV-VIS Spectrophotometer was used to calculate the average peak of the absorption spectra as $\lambda=784 \mathrm{~nm}$. Imaging by Transmission Electron Microscopy (TEM) was used to verify consistency in shape and size. 
2.5. Gold Nanorod Conjugation to Antibody Followed by PEGylation. Gold nanorods were first bioconjugated according to the method presented by Liao and Hafner [14]. Bioconjugation of gold nanorods to antibodies was carried out with the LC-SPDP following the procedure provided. After equilibrating the vial of LC-SPDP reagent to room temperature, $20 \mathrm{mM}$ LC-SPDP was prepared in dimethylsulfoxide, DMSO (Sigma-Aldrich). Cetuximab was used without modification. Twenty-five milliliters of LC-SPDP solution was added to $1 \mathrm{~mL}$ of cetuximab solution and allowed to incubate for $60 \mathrm{~min}$ at room temperature yielding a calculated linker:antibody molar ratio of $37: 1$. The cetuximab mixture was exchanged into $1 \mathrm{~mL}$ of pure PBSEDTA buffer by using a Zeba desalt spin column (Pierce Biotechnology, Rockford, IL) or a Microcon centrifugal filter device (Millipore, Bedford, MA). Reaction byproducts, excess cetuximab, and excess LC-SPDP were also removed by the desalting column. Forty milliliters of raw nanorods were centrifuged (7000 g, $20 \mathrm{~min}$ ), decanted, and then, resuspended in $1 \mathrm{~mL}$ of PBS-EDTA. Two and a half microliters of the antibody/cross-linker mix were added to the nanorod solution and then incubated at room temperature overnight. The nanorods were then PEGylated by adding $10 \mu \mathrm{L}$ of $1 \mathrm{mM}$ mPEG-SH and $100 \mu \mathrm{L}$ of $2 \mathrm{mM}$ potassium carbonate at room temperature and again incubated overnight. Finally, the bioconjugated nanorods were centrifuged, decanted, and resuspended in PBS-EDTA several times to remove excess CTAB and unreacted mPEG-SH [14].

2.6. Antibody Conjugation to PEGylated Gold Nanorods. PEGylated gold nanorods were conjugated to the antibody, cetuximab, using a method similar to Liao and Hafner [14] with one major modification-the order of conjugation and PEGylation. Cetuximab and the LC-SPDP crosslinker were combined in varying molar ratios, desalted, and then added to nanorods that were previously PEGylated. Instead of PEGylating, the conjugated nanorod-crosslinked-antibody unit, the already biocompatible PEGylated nanorod, was crosslinked to the antibody. In our protocol, bioconjugation of gold nanorods to antibodies was carried out with the following modifications. Forty milliliters of raw nanorods were centrifuged $(7000 \mathrm{~g}, 20 \mathrm{~min})$, decanted, and then resuspended in $1 \mathrm{~mL}$ of PBS-EDTA. The nanorods were then PEGylated by adding $10 \mu \mathrm{L}$ of $1 \mathrm{mM}$ mPEG-SH and $100 \mu \mathrm{L}$ of $2 \mathrm{mM}$ potassium carbonate and incubated overnight. The biofunctionalized nanorods were centrifuged, decanted, and resuspended in PBS-EDTA several times to remove excess CTAB and mPEG-SH. Various amounts of the $20 \mathrm{mM}$ crosslinker, LC-SPDP, in DMSO solution were added to $1 \mathrm{~mL}$ of cetuximab and allowed to incubate for $60 \mathrm{~min}$ at room temperature yielding a tunable linker:antibody molar ratio. $100-200 \mu \mathrm{L}$ of the antibody/cross-linker mix was added to the PEGylated nanorods and then incubated at room temperature overnight. Either a Zeba desalt spin column (Pierce Biotechnology, Rockford, IL) or a Microcon centrifugal filter device (Millipore) was used to exchange the buffer for PBS-EDTA and to remove the reaction byproducts of the PEGylated nanorod-antibody complex.
2.7. Order of PEGylation and Conjugation of Nanorod to Antibody Comparison. In Figure 1, we summarize the sequence of the two conjugation protocols that we compare in three steps to clearly illustrate the difference between the processes for gold nanorod conjugation to antibody followed by PEGylation [14] and antibody conjugation to PEGylated gold nanorods.

Figure 1(a) shows the protocol of Liao and Hafner [14] that requires adding the crosslinker to the antibody (step 1), conjugating the antibody to the gold nanorods (step 2) followed by PEGylation (step 3). On the contrary, Figure 1(b) shows our modification of Liao's protocol that describes attaching the crosslinker to the antibody (step 1), PEGylating the nanorod (step 2), then conjugating the antibody to the PEGylated nanorod (step 3). Basically, the difference in the sequence of our protocol and the Liao [14] protocol is that we PEGylate only the nanorods not the nanorod-antibody conjugate.

\subsection{Gold Nanorod to Antibody Conjugation Characterization.} Gold nanorod/antibody conjugations were examined for structure, consistency, and efficiency by TEM. Specifically, we used carbon only copper grids, uranyl acetate stain, and an FEI TecnaiT12 $80 \mathrm{kv}$ or $120 \mathrm{kv}$ (Twin TEM, Hillsboro, OR). We captured digital images of the gold nanorods and conjugates on an AMT (Danvers, MA) 2k camera. UV-VIS Spectrophotometer was used to measure percent transmittance and calculate the plasmon resonance absorption. A modified ELISA assay was used to evaluate the efficiency of antibody binding before and after conjugation to GNRs. We added $500 \mu \mathrm{L}$ PBS to Recombinant Human epidermal growth factor receptor (EGFR)/ErbB1 Fc Chimera, CF $(50 \mu \mathrm{g} / \mathrm{vial}$ R\&D Systems, Inc., Minneapolis, MN) for a final concentration of $0.1 \mathrm{mg} / \mathrm{mL}$ and used $50 \mathrm{ng} / 100 \mu \mathrm{L}$ of rEGFR in PBS $\mathrm{w} / \mathrm{Ca}^{++}$and $\mathrm{Mg}$ to coat a $96-$-well plate. The plate was covered with saran wrap or plate sealer and incubated overnight at $4^{\circ} \mathrm{C}$. We blocked the plate with $1 \%$ BSA in PBS w/o Ca ${ }^{++}$and $\mathrm{Mg}$ for nonspecific binding and incubated for $1 \mathrm{hr}$ RT or stored at 4 degrees until use. The BSA or PBS was removed from wells and the samples were added, incubated for $1 \mathrm{hr}$, and washed with PBS several times. Samples were analyzed by the Pearl Impulse Imager (LI-COR, Lincoln, $\mathrm{NB})$.

2.9. Near Infrared Photothermal Therapy. Head and neck human squamous cell carcinoma, (Thomas Carey, University of Michigan, Ann Arbor, MI), cell line SCC-5, was used in this study. The HNSCC line was maintained in Dulbecco's Modified Eagle Medium containing 10\% fetal bovine serum, supplemented with L-glutamine, penicillin, and streptomycin and incubated at $37^{\circ} \mathrm{C}$ in $5 \% \mathrm{CO}_{2}$. The measured concentration of the PEGylated nanorods was $\mathrm{CONC}_{\mathrm{PEG}-\mathrm{NR}}=$ $4.41 \mathrm{mg} / \mathrm{mL}$. The optical density of the PEGylated nanorods is OD $_{\text {PEG-NR }}=8.24\left(\sim 1.73 \times 10^{12}\right.$ particles $\left./ \mathrm{mL}\right)$. Both PEGylated nanorods and nanorod-antibody conjugates were used with average plasmon resonance absorption of $785 \mathrm{~nm}$. A NIR diode laser (SDL, Inc, San Jose, CA, 8350) was used to 
(1)

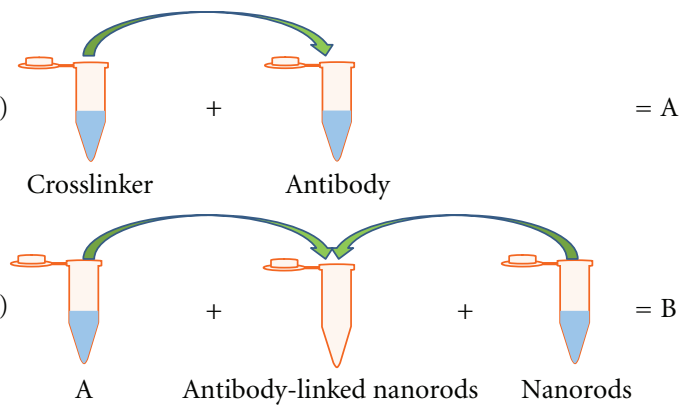

(3)

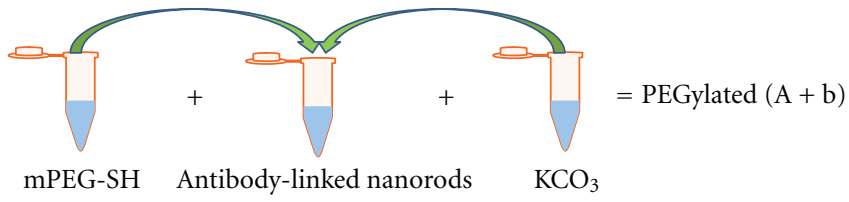

(a) Gold nanorod conjugation to antibody followed by PEGylation

(1)

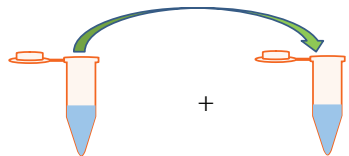

$=\mathrm{A}$

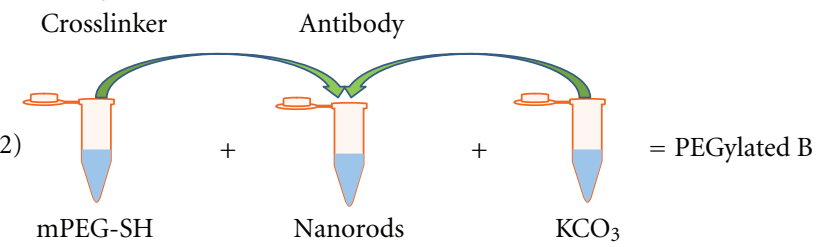

(3)

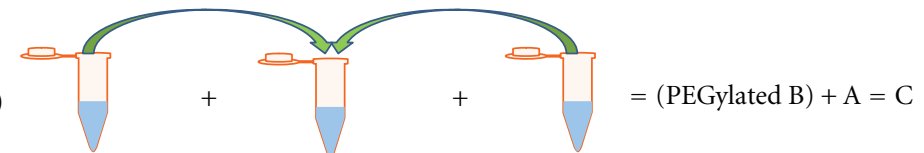

PEGylated nanorods

Crosslinker and antibody

(b) Conjugation of Antibody to PEGylated Gold Nanorod

FIGURE 1: Illustration of Nanorod to Antibody Conjugation. (a) Gold nanorod conjugation to antibody followed by PEGylation and (b) conjugation of antibody to PEGylated nanorod.

perform the photothermal therapy of cells, 4 min exposure time, $785 \mathrm{~nm}$ wavelength, $9.5 \mathrm{~W} / \mathrm{cm}^{2}$ fluence. A cell viability assay was performed with $1: 1$ dilution of Trypan Blue.

\section{Results and Discussion}

In this work, we evaluate a protocol that enhances the targeted treatment of malignant tumors by improving the conjugation efficiency of GNRs to tumor-targeted antibodies. We can consequently improve the delivery of the nanorods making the NIR PTT more effective. By modifying an existing protocol [14], we take advantage of covalent bonds, sulfhydryl and amide group chemistry to improve conjugation efficiency. We compare the order of conjugation and PEGylation and its effects on the nanorods forming clusters, aggregating, and the percentage of binding efficiency of the antibody in both cases. Also we compare the binding efficiency of the antibody before and after conjugation and the therapeutic effect of the nanorods for photothermal therapy before and after conjugation.
3.1. Nanorod Characterization and Biofunctionalization. The first objective in this study was to identify a successful modality to selectively target gold nanorods to malignant cells by optimizing conjugation parameters to the antiEGFR antibody, cetuximab. By conjugating nanorods with antibodies, we can improve the specificity of nanorods. The quality of the conjugation of nanorods to antibodies using the LC-SPDP crosslinker and PEG-SH can be affected by the sequential order of the steps as depicted in Figure 2.

The TEM images in Figure 2 show the physical differences that the order of PEGylation and conjugation, as illustrated in Figure 1, has on a collection of gold nanorods. The results we obtained by repeating the method published by Liao and Hafner [14] using anti-EGFR instead of antiRabbit IgG antibody are shown in Figure 2(a). In short, the conjugated GNRs-antibody unit was PEGylated; this procedure resulted in more than a $70 \%$ loss of nanorod individuality, described here as aggregation. To overcome this aggregation, we conjugated PEGylated GNRs to antibodies, shown in Figure 2(b). We observed more than $70 \%$ of the 


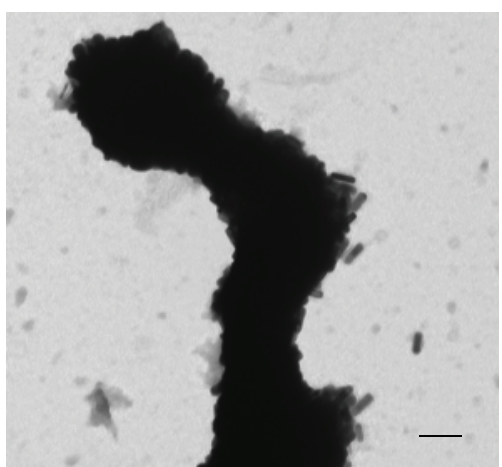

(a)

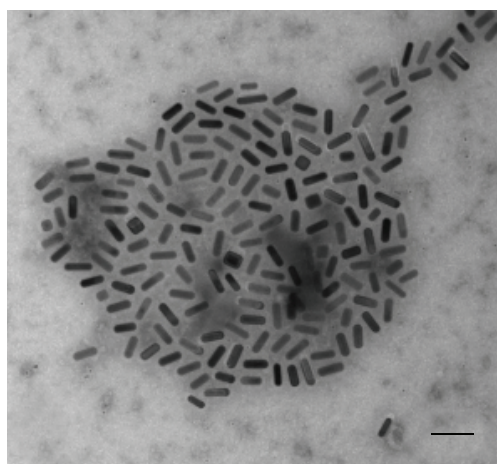

(b)

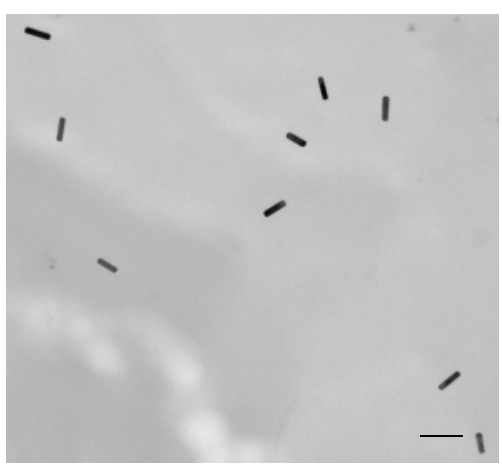

(c)

FIgUre 2: TEM images of nanorod conjugation to antibody before PEGylation versus PEGylated nanorod conjugation to antibody. (a) Nanorods first conjugated to anti-EGFR antibody then PEGylated; (b) Nanorods first PEGylated then conjugated to anti-EGFR antibody; (c) PEGylated nanorods without antibody or crosslinker present. All scale bars shown are $100 \mathrm{~nm}$.

GNRs in clusters of various shapes and sizes. In Figure 2(c), we show PEGylated GNRs with no antibodies or crosslinker, as a control. We do not find aggregation like in Figure 2(a) or clustering like in Figure 2(b) using the same concentration of GNRs. This indicates that the nanorod-antibody interaction is affected by the order of conjugation and PEGylation.

Antibody binding efficiency was also affected by the PEGylation and conjugation order. Using the unconjugated antibody as the binding efficiency standard, we surveyed the binding efficiencies resulting from the protocols illustrated in

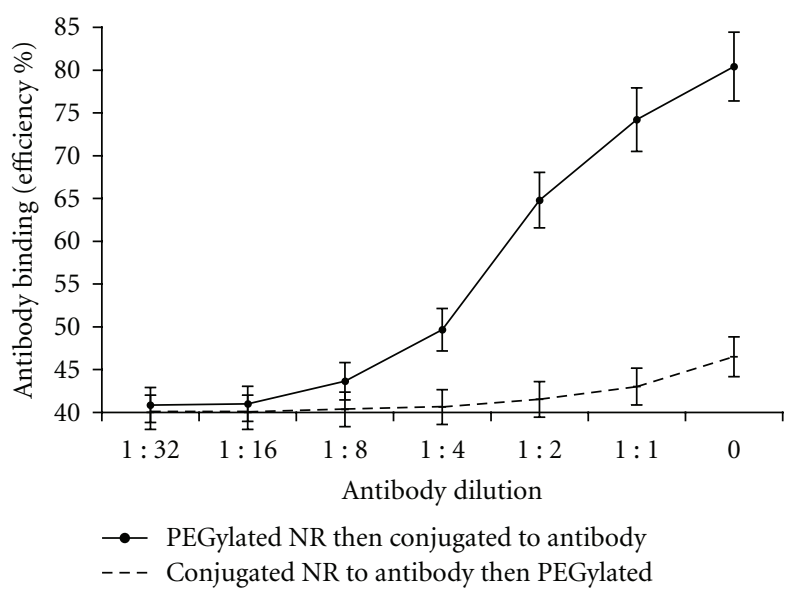

Figure 3: A comparative antibody binding assay based on the sequential order of conjugation and PEGylation, summarizing the binding efficiency of antibodies when conjugated to nanorods then PEGylated (solid line) and antibodies conjugated to PEGylated nanorods (dashed line).

Figure 1 using a modified ELISA assay. In Figure 3, we summarize the results for a titration of antibody concentrations and without any dilution to the antibody concentration.

We observe that the antibody binding efficiency increases as antibody concentration increases in both cases. Without any dilution to the antibody concentration, we measure a $46.5 \%$ binding efficiency for the antibodies conjugated to GNRs then PEGylated using the Liao and Hafner protocol [14] versus a $80.4 \%$ binding efficiency for the antibodies conjugated to PEGylated GNRs. By modifying the order of conjugation and PEGylation, we demonstrate a 6-fold improvement in the binding efficiency of the conjugated antibody.

Our goal was to optimize some parameters of photothermal therapy without compromising the aspects that were already effective, namely, the targeting ability of the antibodies and the therapeutic ability of the nanorods when used in combination with the NIR laser. We measured the binding ability of unconjugated anti-EFGR antibody to the antigen, the epidermal growth factor receptor. Using the unconjugated antibody as the standard of binding efficiency on a scale from 0 to 5 , we studied the binding efficiency of the antibody after being conjugated to PEGylated nanorods, as shown in Figure 4(a).

We found that the binding efficiency slightly improved after conjugation from 3.93 to 4.4 on a scale from 0 to 5 but considering the standard error, we can estimate that the binding efficiency is approximately the same for the antibody before and after conjugation to PEGylated nanorods, Figure 4(a). This binding assay confirms that the antibody is still binding after conjugation. The $9 \%$ difference in binding efficiency of antibody before and after conjugation to nanorods is relatively negligible when compared to the binding efficiency of the antibody that was conjugated to the nanorod then PEGylated which had a binding efficiency of 0.29 , an $82 \%$ difference from the antibody binding before conjugation. 


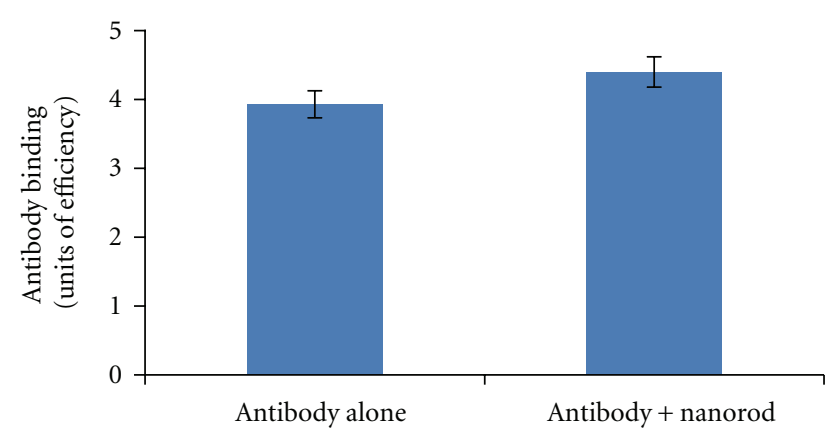

(a) Anti-EGFR antibody binding ability

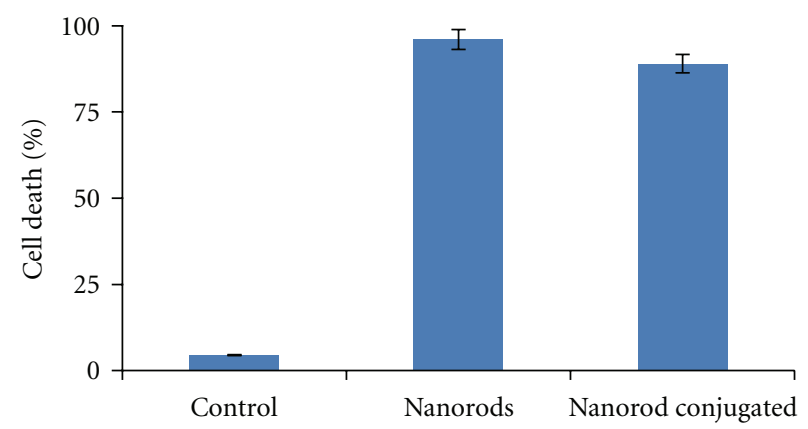

(b) Nanorods therapeutic ability

FIGURE 4: (a) Anti-EGFR antibody binding assay shows the antibody function before and after conjugation to the PEGylated gold nanorod. (b) Cell viability assay summarizes the percentage of cells dead after photothermal therapy with laser only, laser plus PEGylated nanorods, and laser plus nanorods conjugated to the anti-EGFR antibody.

Previous studies report that GNR-mediated NIR PTT provides approximately a $95 \%$ death rate, which is comparable to what we found before and after conjugation. In the process of optimizing the active targeting component by conjugating the GNRs to antibodies, we maintain the therapeutic capability that has been established and achieved with much success. In Figure 4(b), we show the effect that conjugating the nanorods to antibodies has on photothermal therapy. This cell viability assay shows the percentage of dead cells after treatment as performed in previous in vitro studies without washing cells after adding GNRs [4]. The cells were treated with the NIR laser only as a control, with the laser and PEGylated nanorods, and with the laser plus PEGylated nanorods conjugated to the anti-EGFR antibody. The percentages of dead cells after these treatments were $4.5 \%, 96 \%$, and $89 \%$, respectively. There is only a $7 \%$ difference in the therapeutic effect for nanorods before and after the conjugation process which likely attributed to distance between GNRs and the cell surface due to the antibody and crosslinker. All experiments were performed with a $785 \mathrm{~nm}$ diode laser at $9.5 \mathrm{~W} / \mathrm{cm}^{2}, \sim 4$ minutes using GNRs with $\mathrm{OD}_{\text {PEG-NR }}=4.12\left(\sim 8.65 \times 10^{11}\right.$ particles $\left./ \mathrm{mL}\right)$, and $300: 1$ crosslinker : antibody ratio.

The physiological conditions inside the body require a resilient and functional conjugation to ensure optimal delivery of the GNRs to malignant tumors and successful

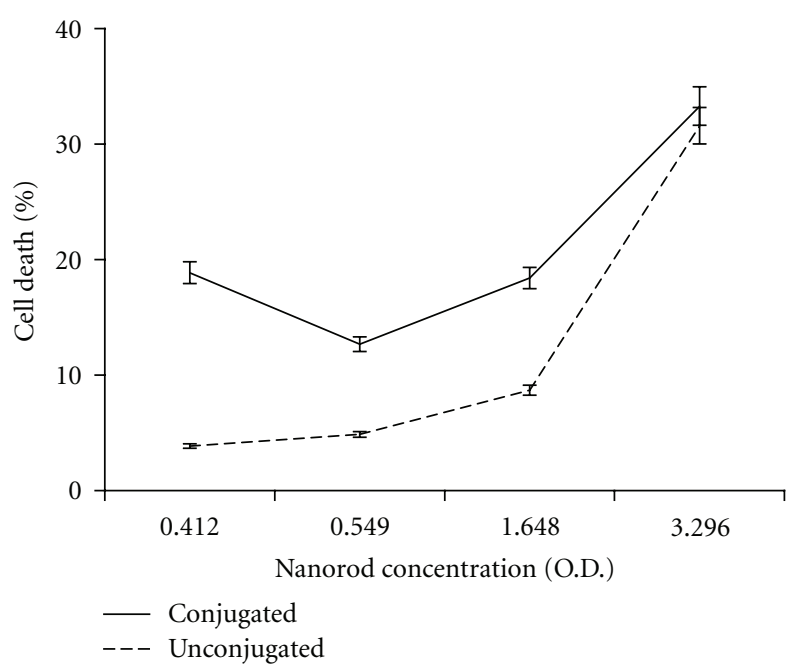

Figure 5: This cell viability assay shows the percentage of cell death of conjugated (solid line) and unconjugated (dashed line) nanorods after photothermal therapy with increasing nanorod concentration after three washes.

treatment upon arrival. We have confirmed that after conjugation, both the antibody binding efficiency and the GNRs therapeutic ability are individually comparable to their performance before conjugation. To further evaluate the conjugation performance and the photothermal effect with GNRs, we treated the cells with GNRs, alone and conjugated, and washed with medium three times to remove any unbound GNRs. We observe that there is some adsorption effect happening with the GNRs on the surface but this is distinguishable at very dilute concentrations of the GNRs. In Figure 5, we compare the percentage of cell death after NIR PTT with GNRs alone and conjugated.

GNRs with optical densities of $0.412,0.549,1.648$, and 3.296 were conjugated to antibody concentrations of 14.3 , $19.1,11.12$, and $9.52 \mu \mathrm{g} / \mathrm{mL}$, respectively. We show a trend that as the nanorod concentration increases, the cell death increases. The difference in the percentage of cell death caused by NIR PTT is observed for lower concentrations of GNRs correlating to higher antibody concentrations. There may also a difference in the binding effects observed for the anti-EGFR antibody binding to the EGFR used in the assay as displayed in Figure 3 and the binding that results from the EGFR expressed on the surface of the SCC- 5 cells used for the cell viability experiment after three washes in Figure 5. The antibody-to-nanorod ratio and resultant relative therapeutic effects and stoichiometry have not been studied here.

\section{Summary and Conclusion}

We have explored optimizing the parameters for the improved success of using covalent bonds to conjugate GNRs to the anti-EGFR antibody, cetuximab. The covalent conjugation of the antibody to GNR facilitates active targeting of the nanorods to the tumor site. This kind of active targeting is more efficient than previously reported 
results [14] that PEGylated the antibody-nanorod conjugate. We characterized the efficiency of the conjugation of the gold nanorods to cetuximab by comparing the antibody binding efficiency and the photothermal effect of nanorods before and after conjugation. The percentage of antibody binding efficiency is 6 -fold (33.9\%) greater when conjugating the antibody to an already PEGylated nanorod versus PEGylating the nanorod-antibody conjugation [14]. We show that binding ability of the conjugated antibody to the epidermal growth factor receptor (EGFR) has not been affected by conjugation, whereas the binding efficiency was 3.93 before conjugation and 4.4 after conjugation on a scale from 0 to 5 .

In addition, cell death by NIR photothermal therapy with the use of gold nanorods is not compromised following conjugation of gold nanorods to an antibody. The percentage of cells living after photothermal treatment with the laser and PEGylated nanorods was only $4 \%$, when compared to the $11 \%$ of cells living after treatment with laser plus nanorods conjugated to the anti-EGFR antibody. The difference in the photothermal therapeutic effect for nanorods before and after the conjugation process is only a $7 \%$. NIR photothermal treatment with the antibody-nanorod conjugate selectively heated the GNR and was sufficient to kill nearly $90 \%$ of tumor cells, which is comparable to photothermal therapy with the GNR alone. These results indicate that nanorodantibody conjugates are promising as an improved active targeting agent for future in vivo studies. Further investigation is needed to determine what is happening with the antibody binding, how many nanorods are binding to each antibody, and what biodistribution improvements pertaining to in vivo studies are available. This combination of active targeting and subsequent photothermal treatment of malignant cells is a viable approach for the treatment of a variety of cancer types that overexpress the epidermal growth factor including head and neck, colorectal, ovarian, cervical, skin, breast, bladder, pancreatic, and prostate cancers.

\section{Acknowledgments}

This work has been funded by the National physical science consortium, NSF Grant: EPS-0814103, and NIH/NIDCR R21 Grant: 1R21DE019232-01A2. Part of this work has been presented at The 2011 International Conference for Nanoparticles and Nanomaterials to Nanodevices and Nanosystems (IC4N). The authors thank the UAB High Resolution Imaging Facility and Melissa Foley for assistance with TEM imaging. They acknowledge Yolanda Hartman, Jason Warram, Kirk Zinn, Renato Camata, Paul Castellanos, and William Grizzle for encouragement and supportive discussions.

\section{References}

[1] L. C. Kennedy, L. R. Bickford, N. A. Lewinski et al., "A new era for cancer treatment: gold-nanoparticle-mediated thermal therapies," Small, vol. 7, no. 2, pp. 169-183, 2011.

[2] X. Huang, I. H. El-Sayed, and M. A. El-Sayed, "Applications of gold nanorods for cancer imaging and photothermal therapy," Methods in Molecular Biology, vol. 624, pp. 343-357, 2010.
[3] C. Loo, A. Lowery, N. Halas, J. West, and R. Drezek, "Immunotargeted nanoshells for integrated cancer imaging and therapy," Nano Letters, vol. 5, no. 4, pp. 709-711, 2005.

[4] X. Huang, I. H. El-Sayed, W. Qian, and M. A. El-Sayed, "Cancer cell imaging and photothermal therapy in the nearinfrared region by using gold nanorods," Journal of the American Chemical Society, vol. 128, no. 6, pp. 2115-2120, 2006.

[5] E. B. Dickerson, E. C. Dreaden, X. Huang et al., "Gold nanorod assisted near-infrared plasmonic photothermal therapy (PPTT) of squamous cell carcinoma in mice," Cancer Letters, vol. 269, no. 1, pp. 57-66, 2008.

[6] C. L. Chen, L. R. Kuo, C. L. Chang et al., "In situ realtime investigation of cancer cell photothermolysis mediated by excited gold nanorod surface plasmons," Biomaterials, vol. 31, no. 14 , pp. 4104-4112, 2010.

[7] J. G. Morton, E. S. Day, N. J. Halas, and J. L. West, "Nanoshells for photothermal cancer therapy," Methods in Molecular Biology, vol. 624, pp. 101-117, 2010.

[8] A. M. Gobin, M. H. Lee, N. J. Halas, W. D. James, R. A. Drezek, and J. L. West, "Near-infrared resonant nanoshells for combined optical imaging and photothermal cancer therapy," Nano Letters, vol. 7, no. 7, pp. 1929-1934, 2007.

[9] W. R. Chen, R. L. Adams, A. K. Higgins, K. E. Bartels, and R. E. Nordquist, "Photothermal effects on murine mammary tumors using indocyanine green and an 808-nm diode laser: an in vivo efficacy study," Cancer Letters, vol. 98, no. 2, pp. 169173, 1996.

[10] X. Huang, P. K. Jain, I. H. El-Sayed, and M. A. El-Sayed, "Plasmonic photothermal therapy (PPTT) using gold nanoparticles," Lasers in Medical Science, vol. 23, no. 3, pp. 217-228, 2008.

[11] I. H. El-Sayed, X. Huang, and M. A. El-Sayed, "Selective laser photo-thermal therapy of epithelial carcinoma using anti-EGFR antibody conjugated gold nanoparticles," Cancer Letters, vol. 239, no. 1, pp. 129-135, 2006.

[12] H. Maeda, K. Greish, and J. Fang, "The EPR effect and polymeric drugs: a paradigm shift for cancer chemotherapy in the 21st century," Advances in Polymer Science, vol. 193, no. 1, pp. 103-121, 2006.

[13] R. K. Jain, "Transport of molecules in the tumor interstitium: a review," Cancer Research, vol. 47, no. 12, pp. 3039-3051, 1987.

[14] H. Liao and J. H. Hafner, "Gold nanorod bioconjugates," Chemistry of Materials, vol. 17, no. 18, pp. 4636-4641, 2005.

[15] T. K. Sau and C. J. Murphy, "Seeded high yield synthesis of short Au nanorods in aqueous solution," Langmuir, vol. 20, no. 15, pp. 6414-6420, 2004.

[16] A. M. Chen, "Current and future applications of immunological attenuation via pegylation of cells and tissue," BioDrugs, vol. 15, no. 12, pp. 833-847, 2001.

[17] L. Shan, Polyethylene Glycol-Coated (PEG5000) Gold Nanoparticles, National Center for Biotechnology Information, Bethesda, Md, USA, 2010. 

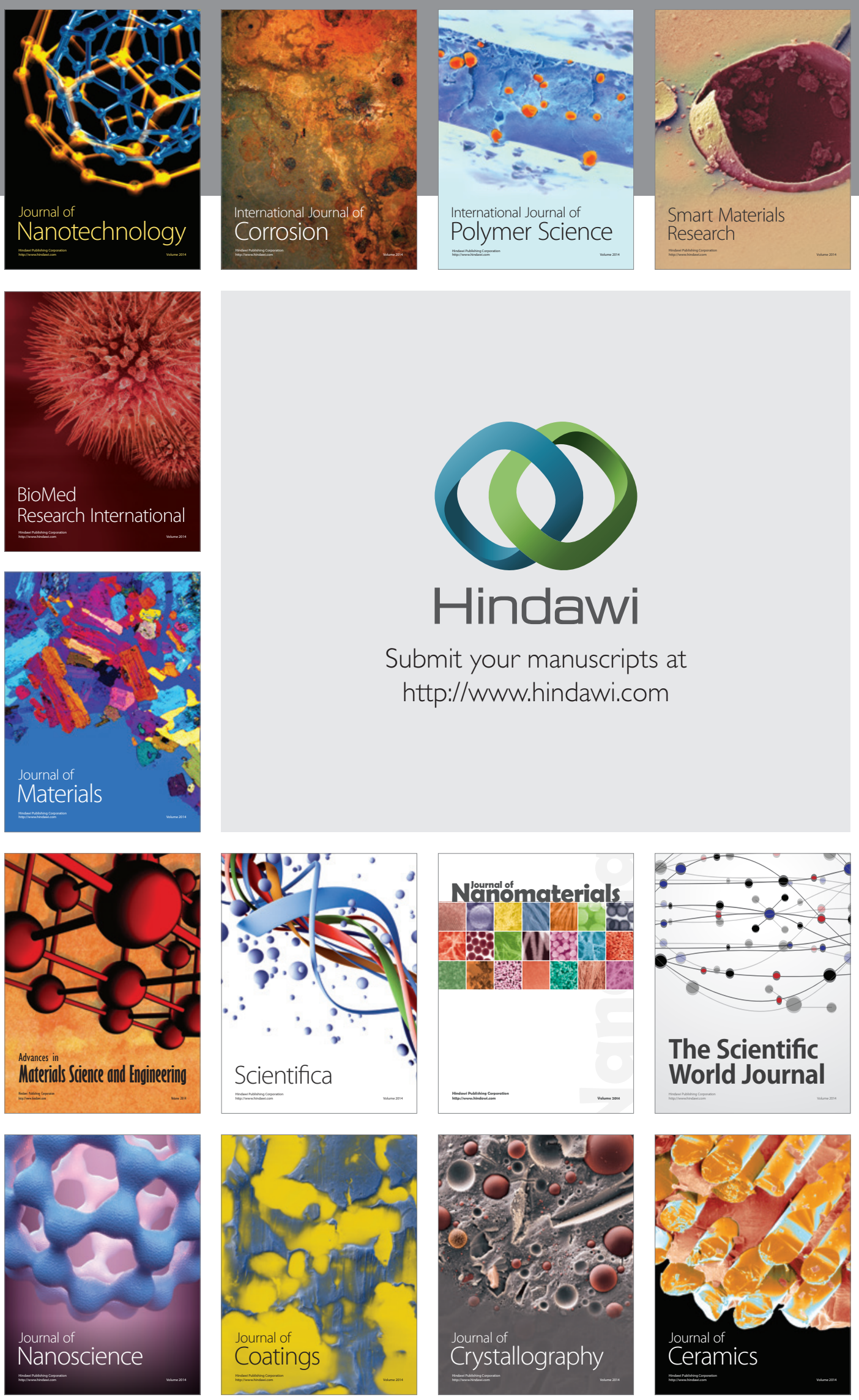

The Scientific World Journal

Submit your manuscripts at

http://www.hindawi.com

\section{World Journal}

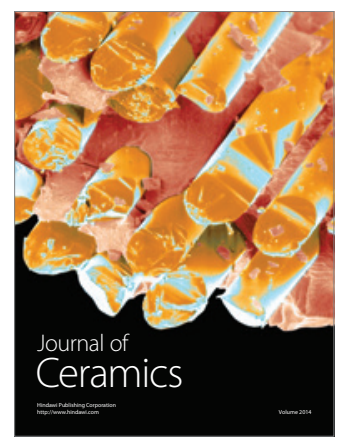

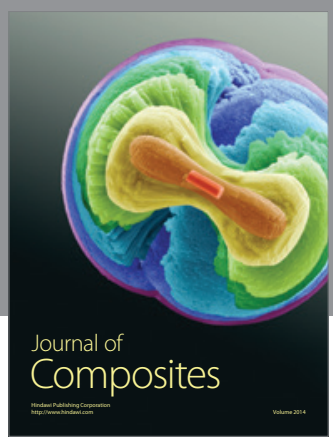
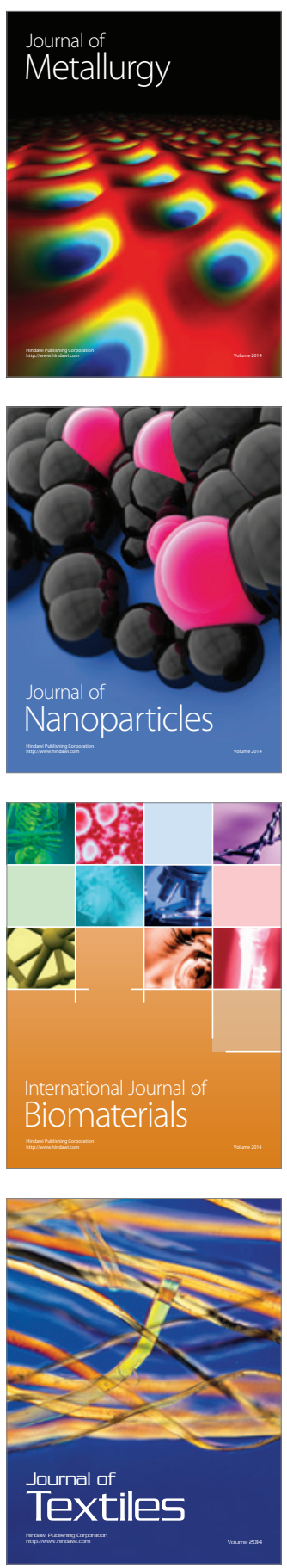